\title{
Outage Capacity Optimization for Free-Space Optical Links With Pointing Errors
}

\author{
Ahmed A. Farid, Student Member, IEEE, and Steve Hranilovic, Member, IEEE
}

\begin{abstract}
We investigate the performance and design of free-space optical (FSO) communication links over slow fading channels from an information theory perspective. A statistical model for the optical intensity fluctuation at the receiver due to the combined effects of atmospheric turbulence and pointing errors is derived. Unlike earlier work, our model considers the effect of beam width, detector size, and jitter variance explicitly. Expressions for the outage probability are derived for a variety of atmospheric conditions. For given weather and misalignment conditions, the beam width is optimized to maximize the channel capacity subject to outage. Large gains in achievable rate are realized versus using a nominal beam width. In light fog, by optimizing the beam width, the achievable rate is increased by $80 \%$ over the nominal beam width at an outage probability of $10^{-5}$. Well-known error control codes are then applied to the channel and shown to realize much of the achievable gains.
\end{abstract}

Index Terms-Atmospheric turbulence, beam misalignment, free-space optical (FSO) communications, optical channel capacity, outage probability, pointing errors.

\section{INTRODUCTION}

$\mathbf{F}$ REE-SPACE optical (FSO) systems are an exciting technology that establish point-to-point communication links through the atmosphere. They provide high security, low cost, low power, and high rates due to the unregulated bandwidth [1]. Such links are suitable for $1-2 \mathrm{~Gb} / \mathrm{s}$ rates over distances in the range of $1-5 \mathrm{~km}$. Optical signal propagation in free space is affected by atmospheric turbulence and pointing errors, which fade the signal at the receiver and deteriorate the link performance. In this paper, we investigate the design and performance of slow-fading FSO channels corrupted by these impairments from an information theory perspective and demonstrate that optimizing the beam width results in large gains in channel capacity.

Atmospheric turbulence causes fluctuations in both the intensity and the phase of the received signal due to variations in the refractive index along the propagation path [2]. Many statistical models have been proposed to describe this fluctuation in both weak and strong fading regimes [3], [4]. In addition, misalignment between the transmitter and receiver due to building sway causes pointing errors that limit the

Manuscript received November 24, 2006; revised March 13, 2007. This work was presented in part at the 2006 IEEE Laser and Electro-Optics Society Annual Meeting, Montreal, QC, Canada, October 29-November 2, 2006.

The authors are with the Department of Electrical and Computer Engineering, McMaster University, Hamilton, ON L8S 4K1, Canada (e-mail: farid@grads.ece.mcmaster.ca; hranilovic@mcmaster.ca).

Color versions of one or more of the figures in this paper are available online at http://ieeexplore.iee.org.

Digital Object Identifier 10.1109/JLT.2007.899174 performance of FSO links. The impact of pointing error (jitter) has been widely investigated for intersatellite space-based FSO links [5]-[7] which operate over ranges of many thousands of kilometers. In these links, the assumption of negligible detector aperture size with respect to the beam width at the receiver is made due to the large distances. The effect of pointing error and atmospheric turbulence has also been considered in terrestrial links of shorter range. Modeling the combined impact of turbulence and jitter, as well as the impact of the bit error rate (BER) of communication systems, has been considered [8], [9]; however, in all cases, the detector size is assumed to be negligible compared to the beam width at the receiver, and only uncoded transmission is considered. Optimization of the beam width in FSO systems to minimize BER has also been investigated using the small detector model [10]; however, a formal design procedure for jointly designing beam width and coding was not presented. Recently, information and coding theory have been applied to FSO channels. The ergodic [11] and outage capacities [12] for a Poisson noise model and a Gaussian noise model [13] have been derived in the absence of misalignment errors. The performance of detection techniques [14], bounds on the pairwise error probability for a variety of coding schemes [15], [16], and low-complexity codes [11] have been considered for FSO channels. However, code rate design and joint optimization with the beam width for a finite detector aperture and pointing jitter has not been considered.

This paper presents a formal method to jointly design the beam width and code rate for FSO channels impaired by turbulence and misalignment induced fading. For given atmospheric and misalignment fading statistics, the channel is engineered by selecting a beam width which maximizes the outage capacity. Unlike previous work, a statistical model for FSO links is derived which models the fading due to atmospheric turbulence and pointing errors considering beam width, pointing error variance, and detector size. Since the channel state varies on the order of millions of symbol intervals, we adopt a slow fading channel model and derive expressions for the outage probability for weak and strong turbulence conditions. A key novelty of this paper is that, unlike previous work, combined consideration of beam width optimization and capacity is used to design FSO systems. For a given outage probability or, equivalently, channel availability, the beam width which maximizes the achievable rate for $\mathrm{ON}-\mathrm{OFF}$ keying (OOK) is selected. It is shown that by selecting the optimum beam width versus a nominal one used in a commercial system gives large increases in achievable rates. Well-known error control codes with appropriate rates and complexity are then applied to the channel and shown to realize a large portion of the promised gains. 


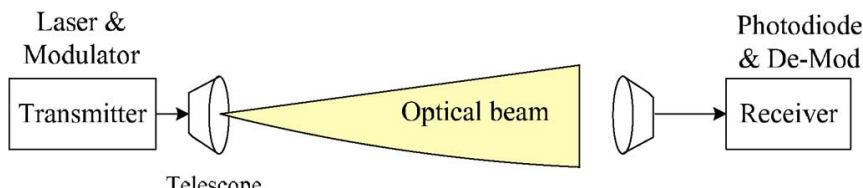

Fig. 1. Block diagram of an FSO link.

A brief description of FSO systems is given in Section II, followed by a discussion on outage capacity. Section III reviews statistical models for the atmospheric turbulence and presents a novel statistical model for pointing errors. The probability of outage is derived in both weak and strong fading regimes, and design criteria are presented in Section IV. Design examples for two weather conditions are presented in Section V. This paper concludes in Section VI with some directions for future work.

\section{System Model And Definitions}

A block diagram of an FSO communication link is presented in Fig. 1. The transmitter modulates data onto the instantaneous intensity of an optical beam. In this paper, we consider intensity modulated direct detection channels using OOK modulation, which is widely employed in practical systems. The received photocurrent signal is related to the incident optical power by the detector responsivity $R$. It is assumed that the receiver integrates the photocurrent for each bit period and removes any constant bias due to background illumination. The received signal $y$ suffers from a fluctuation in signal intensity due to atmospheric turbulence and misalignment, as well as additive noise, and can be well modeled as [14]

$$
y=h R x+n
$$

where $x$ is the transmitted intensity, $h$ is the channel state, $y$ is the resulting electrical signal, and $n$ is signal-independent additive white Gaussian noise with variance $\sigma_{n}^{2}$.

The channel state $h$ models the random attenuation of the propagation channel. In our model, $h$ arises due to three factors: path loss $h_{\ell}$, geometric spread and pointing errors $h_{\mathrm{p}}$, and atmospheric turbulence $h_{\mathrm{a}}$. The channel state can be formulated as

$$
h=h_{\ell} h_{\mathrm{p}} h_{\mathrm{a}} .
$$

Note that $h_{\ell}$ is deterministic, and $h_{\mathrm{p}}$ and $h_{\mathrm{a}}$ are random with distributions discussed in Section III. Since the time scales of these fading processes $\left(\approx 10^{-3}-10^{-2} \mathrm{~s}[14]\right)$ are far larger than the bit interval $\left(\approx 10^{-9} \mathrm{~s}\right), h$ is considered to be constant over a large number of transmitted bits. Notice that the use of interleaving to allow for averaging over a large number of fading states is impractical in this channel. This block fading channel is often termed a slow fading or nonergodic channel [17] in which an $h$ is chosen from the random ensemble according to distribution $f_{h}(h)$ and fixed over a long block of bits.

The transmitted signal is taken as symbols drawn equiprobably from an OOK constellation such that $x \in\left\{0,2 P_{t}\right\}$, and $P_{t}$ is the average transmitted optical power. The received electrical signal-to-noise ratio (SNR) for OOK signaling and a slow fading channel is defined as

$$
\operatorname{SNR}(h)=\frac{2 P_{t}^{2} R^{2} h^{2}}{\sigma_{n}^{2}}
$$

and is random due to the influence of $h$.

\section{A. FSO Channel Capacity and Outage Probability}

Channel capacity is the maximum achievable data rate that can be reliably communicated between the transmitter and the receiver [18]. In this paper, we restrict our attention to the practical case of equiprobable binary OOK alphabets, and capacity refers to the maximum rate using this source distribution.

The channel capacity of time-varying fading channels depends on the information available at the transmitter and/or receiver about the channel (channel state information, CSI, and distribution). For the nonergodic slow-fading channels considered here, we assume that the receiver has perfect knowledge of $h$ and that the transmitter sends information at a rate of $R_{0}$ bits/channel use. The instantaneous capacity corresponding to a channel state $h=h^{\prime}$ for binary OOK signal is given by

$$
\mathcal{C}\left(\operatorname{SNR}\left(h^{\prime}\right)\right)=\int \sum_{x} f_{y \mid x}(y \mid x) p_{x}(x) \log _{2} \frac{f_{y \mid x}(y \mid x)}{f_{y}(y)} d y
$$

where

$$
\begin{aligned}
x & \in\left\{0,2 P_{t}\right\} \\
p_{x}(x=0) & =p_{x}\left(x=2 P_{t}\right)=0.5 \\
f_{y \mid x}(y \mid x) & =\mathcal{N}\left(h^{\prime} R x, \sigma_{n}^{2}\right) \\
f_{y}(y) & =\sum_{x} p_{x}(x) f_{y \mid x}(y \mid x)
\end{aligned}
$$

and $\mathcal{N}\left(\mu, \sigma_{n}^{2}\right)$ denotes a Gaussian distribution with mean $\mu$ and variance $\sigma_{n}^{2}$. Note that SNR is random and depends on the channel state $h$ via (3). Since the channel is random and fixed for a long period of time, there is finite probability that $\mathcal{C}$ is not sufficient to support $R_{0}$. This event is termed an outage [17], and in this case, the transmitted codewords cannot be reliably decoded at the receiver. An appropriate measure of the capacity in this case is the outage probability at rate $R_{0}$, which is defined as

$$
P_{\text {out }}\left(R_{0}\right)=\operatorname{Prob}\left(\mathcal{C}(\operatorname{SNR}(h))<R_{0}\right) .
$$

Equivalently, since $\mathcal{C}(\cdot)$ is monotonically increasing in SNR

$$
P_{\text {out }}\left(R_{0}\right)=\operatorname{Prob}\left(\operatorname{SNR}(h)<\mathcal{C}^{-1}\left(R_{0}\right)\right) .
$$

For these slow-fading FSO channels, there is a tradeoff between $R_{0}$ and $P_{\text {out }}$, which is a critical issue in design. In order to quantify this tradeoff, in the following sections, the distribution of $h$ due to atmospheric turbulence and pointing errors is derived, and $P_{\text {out }}$ is computed for a variety of weather conditions. 


\section{Optical Channel Fading Model}

\section{A. Atmospheric Statistical Models}

Many statistical models for the intensity fluctuation through FSO channels have been proposed over the last two decades [3], [4], [19]. For weak turbulence, the intensity fluctuation probability density function (pdf) is modeled as a log-normal distribution, which has been validated through experimental measurements [2], [4], [14]. The log-amplitude of the optical intensity has a Gaussian pdf with log-amplitude variance $\sigma_{X}^{2}$ given by [2]

$$
\sigma_{X}^{2}=0.30545 k^{7 / 6} C_{n}^{2}(L) z^{11 / 6} \approx \frac{\sigma_{\mathrm{R}}^{2}}{4}
$$

where $C_{n}^{2}(L)$ is the index of refraction structure parameter at altitude $L$ (assumed to be constant along the propagation path), $k=2 \pi / \lambda$ is the optical wavenumber, $z$ is the propagation distance, and $\sigma_{\mathrm{R}}^{2}$ is the Rytov variance defined as [2], [14]

$$
\sigma_{\mathrm{R}}^{2}=1.23 C_{n}^{2} k^{7 / 6} z^{11 / 6} .
$$

Note that $\sigma_{\mathrm{R}}^{2}$ can be measured directly from atmospheric parameters. The intensity distribution is given by

$$
f_{h_{\mathrm{a}}}\left(h_{\mathrm{a}}\right)=\frac{1}{2 h_{\mathrm{a}} \sqrt{2 \pi \sigma_{X}^{2}}} \exp \left(\frac{\left(\ln h_{\mathrm{a}}+2 \sigma_{X}^{2}\right)^{2}}{8 \sigma_{X}^{2}}\right) .
$$

The log-normal distribution cannot characterize scintillation effects in strong turbulence regimes [4], [20]. In a recent approach to FSO channel modeling [3], [4], a Gamma-Gamma distribution was used to model atmospheric fading. In this case, the pdf of $h_{\mathrm{a}}$ is given as

$f_{h_{\mathrm{a}}}\left(h_{\mathrm{a}}\right)=\frac{2(\alpha \beta)^{(\alpha+\beta) / 2}}{\Gamma(\alpha) \Gamma(\beta)}\left(h_{\mathrm{a}}\right)^{\frac{(\alpha+\beta)}{2}-1} K_{\alpha-\beta}\left(2 \sqrt{\alpha \beta h_{\mathrm{a}}}\right)$

where $K_{\alpha-\beta}(\cdot)$ is the modified Bessel function of the second kind, and $1 / \beta$ and $1 / \alpha$ are the variances of the small and large scale eddies, respectively [4]. It was shown that the Gamma-Gamma pdf is in close agreement with measurements under a variety of turbulence conditions [3], [4].

\section{B. Atmospheric Attenuation}

The attenuation of laser power through the atmosphere is described by the exponential Beers-Lambert Law as

$$
h_{\ell}(z)=\frac{P(z)}{P(0)}=\exp (-\sigma z)
$$

where $h_{\ell}(z)$ is the loss over a propagation path of length $z$, $P(z)$ is the laser power at distance $z$, and $\sigma$ is the attenuation coefficient [21]. The attenuation $h_{\ell}$ is considered as a fixed scaling factor during a long period of time, and no randomness exists in its behavior. It depends on the size and distribution of the scattering particles and the wavelength utilized. It can be expressed in terms of the visibility, which can be measured directly from the atmosphere [22], [23]. There is a strong inverse correlation between the turbulence strength and attenuation. For

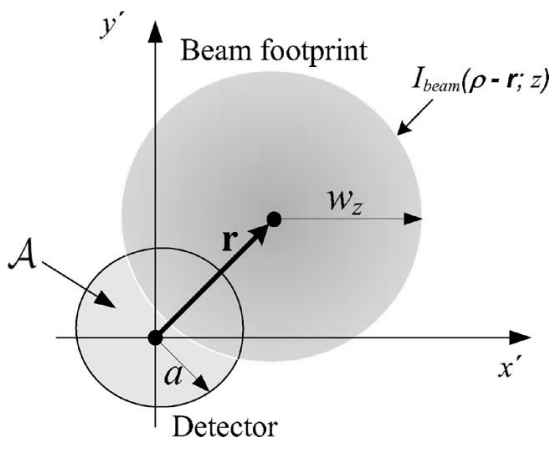

Fig. 2. Detector and beam footprint with misalignment on the detector plane.

example, strong turbulence is highly unlikely to occur during a fog event [24].

\section{Pointing Error}

In line-of-sight FSO communication links, pointing accuracy is an important issue in determining link performance and reliability. However, wind loads and thermal expansions result in random building sways, which, in turn, cause pointing errors and signal fading at the receiver [8]. In this section, we derive a new statistical model for pointing error loss due to misalignment, which considers detector aperture size, beam width, and jitter variance.

For a Gaussian beam, the normalized spatial distribution of the transmitted intensity at distance $z$ from the transmitter is given by [25]

$$
I_{\text {beam }}(\boldsymbol{\rho} ; z)=\frac{2}{\pi w_{z}^{2}} \exp \left(-\frac{2\|\boldsymbol{\rho}\|^{2}}{w_{z}^{2}}\right)
$$

where $\boldsymbol{\rho}$ is the radial vector from the beam center, and $w_{z}$ is the beam waist (radius calculated at $e^{-2}$ ) at distance $z$. The beam waist $w_{z}$ of a Gaussian beam propagating in atmospheric turbulence can be approximated as [26]

$$
w_{z} \approx w_{o}\left[1+\varepsilon\left(\frac{\lambda z}{\pi w_{o}^{2}}\right)^{2}\right]^{\frac{1}{2}}
$$

where $w_{o}$ is the beam waist at $z=0, \varepsilon=\left(1+2 w_{o}^{2} / \rho_{o}^{2}(z)\right)$, and $\rho_{o}(z)=\left(0.55 C_{n}^{2} k^{2} z\right)^{-3 / 5}$ is the coherence length.

Consider a circular detection aperture of radius $a$ and a Gaussian beam profile at the receiver $I_{\text {beam }}$, as shown in Fig. 2 . The attenuation due to geometric spread with pointing error $r$ is expressed as

$$
h_{\mathrm{p}}(\boldsymbol{r} ; z)=\int_{\mathcal{A}} I_{\text {beam }}(\boldsymbol{\rho}-\boldsymbol{r} ; z) d \boldsymbol{\rho}
$$

where $h_{\mathrm{p}}(\cdot)$ represents the fraction of the power collected by the detector, and $\mathcal{A}$ is the detector area. When a pointing error of $\boldsymbol{r}$ is present, $h_{\mathrm{p}}$ is a function of the radial displacement and angle. Due to the symmetry of the beam shape and the detector area, the resultant $h_{\mathrm{p}}(\boldsymbol{r} ; z)$ depends only on the radial distance $r=\|\boldsymbol{r}\|$. Therefore, without loss of generality, we can assume that the radial distance is located along the $x^{\prime}$-axis. The fraction 
TABLE I

NMSE BETWEEN EXACT AND APPROXIMATE $h_{\mathrm{p}}$ EXPRESSIONS

\begin{tabular}{ccccccc}
\hline$w_{z} / a$ & 2 & 4 & 6 & 8 & 10 & 12 \\
\hline $\operatorname{NMSE}\left(\times 10^{-3}\right)$ & 13 & 2.25 & 0.8158 & 0.344 & 0.159 & 0.153
\end{tabular}

of the collected power at a receiver of radius $a$ in the transverse plane of the incident wave can be expressed as

$$
h_{\mathrm{p}}(r ; z)=\int_{-a}^{a} \int_{-\zeta}^{\zeta} \frac{2}{\pi w_{z}^{2}} \exp \left(-2 \frac{\left(x^{\prime}-r\right)^{2}+y^{\prime 2}}{w_{z}^{2}}\right) d y^{\prime} d x^{\prime}
$$

where $\zeta=\sqrt{a^{2}-x^{\prime 2}}$. As shown in the Appendix, this integration can be approximated as the Gaussian form

$$
h_{\mathrm{p}}(r ; z) \approx A_{0} \exp \left(-\frac{2 r^{2}}{w_{z_{\mathrm{eq}}}^{2}}\right)
$$

where $v=(\sqrt{\pi} a) /\left(\sqrt{2} w_{z}\right)$, and

$$
A_{0}=[\operatorname{erf}(v)]^{2}, \quad w_{z_{\mathrm{eq}}}^{2}=w_{z}^{2} \frac{\sqrt{\pi} \operatorname{erf}(v)}{2 v \exp \left(-v^{2}\right)} .
$$

Notice that $A_{0}$ is the fraction of the collected power at $r=0$, and $w_{z_{\text {eq }}}$ is the equivalent beam width. The normalized meansquared error (NMSE) between the exact and the approximate expressions for $h_{\mathrm{p}}$ is given in Table I for different values of $w_{z} / a$. The proposed approximation is in good agreement with the exact value when $w_{z} / a>6$, i.e., NMSE $<10^{-3}$. Fig. 6 in the Appendix plots (8) and (9) versus $r$ for various $w_{z} / a$ values to show their close agreement.

In order to relate this paper to previous work where it is assumed that $w_{z} \gg a$, consider the limiting expression for $h_{\mathrm{p}}$ as $w_{z} / a \rightarrow \infty$, which results in

$$
\lim _{w_{z} / a \rightarrow \infty} h_{\mathrm{p}}(r ; z)=\pi a^{2} I_{\text {beam }}(\boldsymbol{r} ; z)
$$

which is the multiplication of the sampled Gaussian beam at point $r$ and the detector area, as used in previous work.

Consider independent identical Gaussian distributions for the elevation and the horizontal displacement (sway), as was done in previous work [8]. The radial displacement $r$ at the receiver is modeled by a Rayleigh distribution

$$
f_{r}(r)=\frac{r}{\sigma_{s}^{2}} \exp \left(-\frac{r^{2}}{2 \sigma_{s}^{2}}\right), \quad r>0
$$

where $\sigma_{s}^{2}$ is the jitter variance at the receiver. Combining (9) and (10), the probability distribution of $h_{\mathrm{p}}$ can be expressed as

$$
f_{h_{\mathrm{p}}}\left(h_{\mathrm{p}}\right)=\frac{\gamma^{2}}{A_{0}^{\gamma^{2}}} h_{\mathrm{p}}^{\gamma^{2}-1}, \quad 0 \leq h_{\mathrm{p}} \leq A_{0}
$$

where $\gamma=w_{z_{\mathrm{eq}}} / 2 \sigma_{s}$ is the ratio between the equivalent beam radius at the receiver and the pointing error displacement standard deviation at the receiver. Note that it is possible to consider other distributions for the jitter, and the proposed expression for $h_{\mathrm{p}}$ is a general frame work for channel modeling.

\section{Channel Statistical Model}

The probability distribution of $h=h_{\ell} h_{\mathrm{a}} h_{\mathrm{p}}$ can be expressed as

$$
f_{h}\left(h ; w_{z}\right)=\int f_{h \mid h_{\mathrm{a}}}\left(h \mid h_{\mathrm{a}}\right) f_{h_{\mathrm{a}}}\left(h_{\mathrm{a}}\right) d h_{\mathrm{a}}
$$

where $f_{h}\left(h ; w_{z}\right)$ is a family of pdfs parameterized by the beam width $w_{z}$, and $f_{h \mid h_{\mathrm{a}}}\left(h \mid h_{\mathrm{a}}\right)$ is the conditional probability given a turbulence state $h_{\mathrm{a}}$. Recall that $h_{\ell}$ is deterministic and acts as a scaling factor. The resulting conditional distribution can be expressed as

$$
\begin{aligned}
f_{h \mid h_{\mathrm{a}}}\left(h \mid h_{\mathrm{a}}\right) & =\frac{1}{h_{\mathrm{a}} h_{\ell}} f_{h_{\mathrm{p}}}\left(\frac{h}{h_{\mathrm{a}} h_{\ell}}\right) \\
& =\frac{\gamma^{2}}{A_{0}^{\gamma^{2}} h_{\mathrm{a}} h_{\ell}}\left(\frac{h}{h_{\mathrm{a}} h_{\ell}}\right)^{\gamma^{2}-1}, \quad 0 \leq h \leq A_{0} h_{\mathrm{a}} h_{\ell} .
\end{aligned}
$$

Substituting (13) into (12) gives

$$
f_{h}\left(h ; w_{z}\right)=\frac{\gamma^{2}}{\left(A_{0} h_{\ell}\right)^{\gamma^{2}}} h^{\gamma^{2}-1} \int_{h / A_{0} h_{\ell}}^{\infty} h_{\mathrm{a}}^{-\gamma^{2}} f_{h_{\mathrm{a}}}\left(h_{\mathrm{a}}\right) d h_{\mathrm{a}} .
$$

The channel state distribution $f_{h}\left(h ; w_{z}\right)$ can now be computed by substituting proper models for atmospheric turbulence, $f_{h_{\mathrm{a}}}\left(h_{\mathrm{a}}\right)$ into (14). For weak turbulence $\left(\sigma_{\mathrm{R}}^{2}<0.3\right)$, $f_{h_{\mathrm{a}}}\left(h_{\mathrm{a}}\right)$ has a log-normal distribution (5). Substituting into (14) gives

$$
\begin{aligned}
f_{h}(h)= & \frac{\gamma^{2}}{\left(A_{0} h_{\ell}\right)^{\gamma^{2}}} h^{\gamma^{2}-1} \\
& \times \int_{h / A_{0} h_{\ell}}^{\infty} h_{\mathrm{a}}^{-\gamma^{2}} \frac{1}{2 h_{\mathrm{a}} \sqrt{2 \pi \sigma_{X}^{2}}} \exp \left(\frac{\left(\ln h_{\mathrm{a}}+2 \sigma_{X}^{2}\right)^{2}}{8 \sigma_{X}^{2}}\right) d h_{\mathrm{a}} .
\end{aligned}
$$

Simplifying and defining $\mu=2 \sigma_{X}^{2}\left(1+2 \gamma^{2}\right)$ results in

$$
\begin{aligned}
f_{h}\left(h ; w_{z}\right)=\frac{\gamma^{2}}{2\left(A_{0} h_{\ell}\right) \gamma^{2}} h^{\gamma^{2}-1} & \\
& \times \operatorname{erfc}\left(\frac{\ln \left(\frac{h}{A_{0} h_{\ell}}\right)+\mu}{\sqrt{8} \sigma_{X}}\right) e^{\left(2 \sigma_{X}^{2} \gamma^{2}\left(1+\gamma^{2}\right)\right)} .
\end{aligned}
$$

In a strong turbulence regime, $f_{h_{\mathrm{a}}}$ is a Gamma-Gamma distribution, and substituting (6) into (14) results in

$$
\begin{aligned}
f_{h}\left(h ; w_{z}\right)= & \frac{2 \gamma^{2}(\alpha \beta)^{(\alpha+\beta) / 2}}{\left(A_{0} h_{\ell}\right)^{\gamma^{2}} \Gamma(\alpha) \Gamma(\beta)} h^{\gamma^{2}-1} \\
& \times \int_{h / A_{0} h_{\ell}}^{\infty} h_{\mathrm{a}}^{(\alpha+\beta) / 2-1-\gamma^{2}} \mathrm{~K}_{\alpha-\beta}\left(2 \sqrt{\alpha \beta h_{\mathrm{a}}}\right) d h_{\mathrm{a}} .
\end{aligned}
$$


This integration can be expanded into a complex expression of hypergeometric functions. However, these results are not presented in this paper, and efficient numerical techniques are utilized to compute this integral.

\section{Outage AND Design CRiteria}

In this section, we present design criteria based on the derived statistical model of pointing and atmospheric fading to optimize FSO link performance. In all cases, it is assumed that the transmitter is operating at a fixed rate $R_{0} \in[0,1]$ bits/channel use using OOK modulation with given $P_{t}, R, \sigma_{n}^{2}$, $\sigma_{s}^{2}$, and weather conditions. Thus, channel optimization is done only over the beam width.

\section{A. Probability of Outage}

The probability of outage for a slow-fading FSO system under weak and strong turbulence conditions for binary OOK signaling can be computed using $f_{h}(h)$. Combining (4) and (3), the probability of outage at a given rate $R_{0}$ can be expressed as follows:

$$
P_{\text {out }}\left(R_{0}\right)=\operatorname{Prob}\left(\frac{2 P_{t}^{2} R^{2} h^{2}}{\sigma_{n}^{2}}<\mathcal{C}^{-1}\left(R_{0}\right)\right) .
$$

Defining $h_{0}=\sqrt{\mathcal{C}^{-1}\left(R_{0}\right) \sigma_{n}^{2} / 2 P_{t}^{2} R^{2}}$ allows the above expression to be simplified as

$$
P_{\text {out }}\left(R_{0}\right)=\operatorname{Prob}\left(h<h_{0}\right) .
$$

Therefore, the probability of outage is the cumulative density function of $h$ evaluated at $h_{0}$ and is expressed as

$$
P_{\text {out }}\left(R_{0} ; w_{z}\right)=\int_{0}^{h_{0}} f_{h}\left(h ; w_{z}\right) d h .
$$

Notice that $P_{\text {out }}$ is parameterized by the choice of beam width $w_{z}$ through the statistical model for $f_{h}(\cdot)$ derived in (14).

Under weak turbulence conditions, a closed-form expression for $P_{\text {out }}\left(R_{0}\right)$ as a function of $P_{t}, \sigma_{n}^{2}$ and $\sigma_{X}^{2}$ can be obtained using the identity [27, Sec. 3.2]

$$
\int e^{b u} \operatorname{erfc}(a u) d u=\frac{1}{b}\left[e^{b u} \operatorname{erfc}(a u)-e^{\frac{b^{2}}{4 a^{2}}} \operatorname{erf}\left(\frac{b}{2 a}-a u\right)\right]
$$

to yield

$$
\begin{aligned}
& P_{\text {out }}\left(R_{0} ; w_{z}\right) \\
& \quad=\frac{1}{2}\left[e^{\gamma^{2} \psi-2 \sigma_{X}^{2} \gamma^{4}} \operatorname{erfc}\left(\frac{\psi}{\sqrt{8} \sigma_{X}}\right)+\operatorname{erfc}\left(\frac{4 \sigma_{X}^{2} \gamma^{2}-\psi}{\sqrt{8} \sigma_{X}}\right)\right]
\end{aligned}
$$

where $\psi=\ln \left(h_{0} / A_{0} h_{\ell}\right)+\mu$.

For the strong turbulence regime, $f_{h}(h)$ in (15) is substituted into (17), and $P_{\text {out }}\left(R_{0}\right)$ is computed numerically.
TABLE II

WEATHER PARAMETERS

\begin{tabular}{cccccc}
\hline Condition & $\begin{array}{c}C_{n}^{2} \\
\times 10^{-14}\end{array}$ & $\begin{array}{c}\text { Visibility } \\
\mathrm{km}\end{array}$ & $\sigma_{R}^{2}$ & $h_{\ell}$ & $\begin{array}{c}\text { Attenuation } \\
\mathrm{dB} / \mathrm{km}\end{array}$ \\
\hline Clear & 5 & 10 & 1 & 0.9 & 0.44 \\
Light fog & 0.5 & 0.5 & 0.1 & 0.008 & 20 \\
\hline
\end{tabular}

\section{B. FSO Link Design Criteria}

For a given FSO channel, the design of the communication system requires the selection of a transmit beam divergence, as well as a code rate. The fundamental design criterion followed in this paper is to select the beam width $w_{z}^{\text {opt }}$, which maximizes the Shannon channel capacity subject to outage. Notice that the BER is not a parameter in this criterion. A rate $R_{0}$ is termed achievable if there exists a family of codes of code rate $R_{0}$ which can realize any arbitrarily small probability of error. Of course, practical fixed length codes will have a nonzero probability of error; however, good finite length codes approaching Shannon's capacity have been found. Thus, our goal is to first engineer the channel to have a high capacity through optimizing $w_{z}$ and then to apply error-correcting codes to approach these information-theoretic limits.

There exists a tradeoff between the achievable code rate $R_{0}$ and the corresponding probability of outage $P_{\text {out }}\left(R_{0} ; w_{z}\right)$ formalized in (17). In fact, this tradeoff is parameterized by the beam width $w_{z}$ through the statistical fading model in (14). For a given $R_{0}, w_{z}$ can be selected to minimize $P_{\text {out }}$. Alternatively, for a required $P_{\text {out }}$ to be satisfied by the system, the optimum beam width is the one that maximizes the achievable code rate $R_{0}$ that can be transmitted reliably over the channel.

Even after beam optimization, however, not all pairs of $\left(P_{\text {out }}, R_{0}\right)$ are achievable. In this paper, we define the unachievable region as the set of pairs $\left(P_{\text {out }}, R_{0}\right)$ for which it is impossible to find reliable codes. The boundary of this region quantifies the optimum tradeoff between $P_{\text {out }}$ and $R_{0}$ and can be approached by utilizing the optimum beam widths and good error-correcting codes. Note that $w_{z}^{\text {opt }} / a$ is in general different for each point on the optimum tradeoff curve between $P_{\text {out }}$ and $R_{0}$.

In the following section, examples of the application of this information theory-based criterion to FSO channel design are presented for different weather conditions.

\section{EXAMPLES}

\section{A. Weather and System Parameters}

The effect of weather conditions on optical link performance can be characterized by two parameters: the index of refraction structure parameter $C_{n}^{2}$ and $h_{\ell}$. Experimental measurements show that $C_{n}^{2}$ varies from $10^{-15}$ to $2 \times 10^{-13}$ as the turbulence strength varies from weak to strong conditions, and the attenuation factor is empirically expressed in terms of visibility [22]. Table II summarizes different weather conditions and associated values for $C_{n}^{2}$ and visibility. The corresponding Rytov variance $\sigma_{\mathrm{R}}^{2}$ and $h_{\ell}$ for a propagation path of $1 \mathrm{~km} \mathrm{[22]} \mathrm{are}$ also presented. Two cases are considered: clear weather where 
TABLE III

SYSTEM CONFIGURATION

\begin{tabular}{lcc}
\hline Parameter & Symbol & Value \\
\hline Transmission rate & Rate & $1 \mathrm{Gbps}$ \\
Optical transmitted power & $P_{t}$ & $40 \mathrm{~mW}(16 \mathrm{dBm})$ \\
Transmitter/Receiver optics efficiency & $\eta_{T}=\eta_{R}$ & 0.8 \\
Responsivity & $R$ & 0.5 \\
Receiver sensitivity (BER $\left.=10^{-10}\right)$ & $S_{v}$ & $-30 \mathrm{dBm}$ \\
Noise standard deviation & $\sigma_{n}$ & $10^{-7} \mathrm{~A} / \mathrm{Hz}$ \\
Distance between Tx and Rx & $z$ & $1 \mathrm{~km}$ \\
Receiver diameter & $2 a$ & $20 \mathrm{~cm}$ \\
Transmit divergence at $1 / e^{2}$ & $\theta_{T}$ & $2.5 \mathrm{mrad}$ \\
Corresponding Beam radius at $1 \mathrm{~km}$ & $w_{z}$ & $\cong 2.5 \mathrm{~m}$ \\
Pointing errors & $\theta_{s}$ & $1 \mathrm{mrad}$ \\
Corresponding jitter standard deviation & $\sigma_{s}$ & $\cong 30 \mathrm{~cm}$
\end{tabular}

$\sigma_{\mathrm{R}}^{2}=1$, and light fog, where $\sigma_{\mathrm{R}}^{2}=0.1$, which corresponds to strong and weak turbulence, respectively.

The parameters of the system under investigation are presented in Table III [24], [28]. Typical values for receiver sensitivity $S_{v}$ and noise standard deviation $\sigma_{n}$ are taken from a commercial transimpedance amplifier [29]. The transmitter and receiver optics efficiencies $\eta_{T}$ and $\eta_{\mathrm{R}}$ are scaling factors for the received optical power. The nominal values for the normalized beam width and the normalized jitter standard deviation are $w_{z} / a=25$ and $\sigma_{s} / a=3$, respectively [28] and are presented in Table III.

In all simulations, the normalized beam width is restricted to the range $w_{z} / a=5, \ldots, 25$ in discrete steps of $\Delta w_{z} / a=0.1$. For the system parameters in Table III, each step of $\Delta w_{z} / a$ corresponds to a change of approximately $0.01 \mathrm{mrad}$ in transmitter beam divergence half angle, which is well within the alignment tolerance of a practical adaptive beam system. The optimum beam width $w_{z}^{\mathrm{opt}}$ was found for each case through an exhaustive search over this discrete set.

\section{B. Probability of Outage and Link Availability}

In this section, $w_{z}$ is chosen to minimize $P_{\text {out }}$ over $P_{t}$ when the transmitter is constrained to have a fixed code rate $R_{0}$. Qualitatively, beam width optimization balances the impact of $h_{\mathrm{p}}$ and $h_{\mathrm{a}}$ factors on the probability of outage. Widening the beam mitigates pointing errors at the expense of received power while narrowing the beam limits the geometric loss but increases the impact of misalignment.

The probability of outage versus $P_{t}$ for clear weather is shown in Fig. 3 for a code rate $R_{0}=0.5$ (bits/channel use) and beam widths $w_{z} / a=5, \ldots, 25$. The fading distribution for strong turbulence in (15) is applied via numerical integration. For a given $P_{t}$, the optimum beam width $w_{z}^{\text {opt }}$ is selected to minimize $P_{\text {out }}\left(0.5 ; w_{z}\right)$. Notice that optimum beam width increases slowly as a function of $P_{t}$. Intuitively, in this strong turbulence regime, the sensitivity to misalignment fading is low, and increases in $P_{t}$ are used to combat atmospheric fading. A link margin of $\approx 5-\mathrm{dB}$ optical power is realized by optimizing the beam width when the system is designed to satisfy $P_{\text {out }}=10^{-6}$.

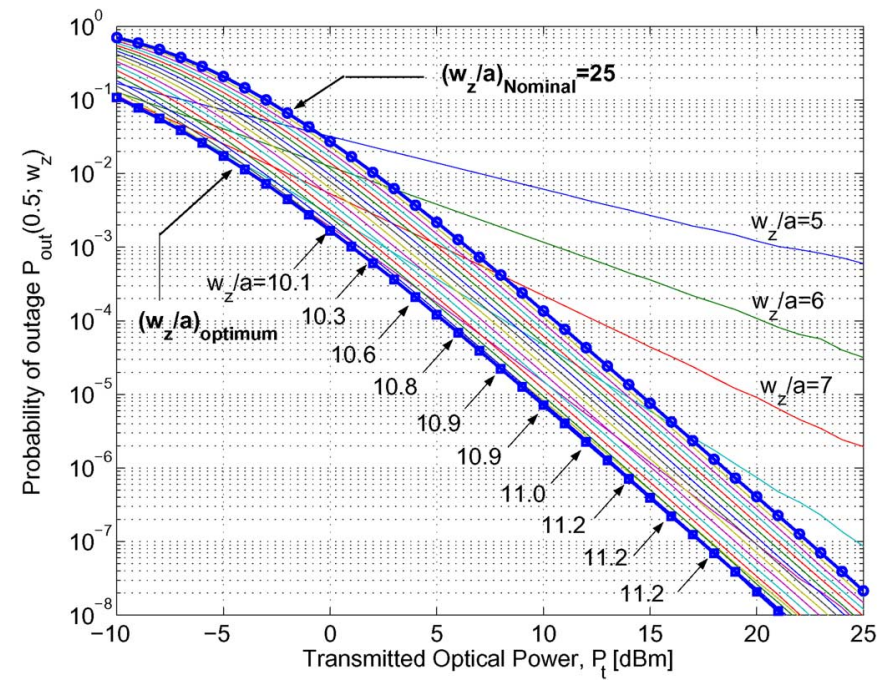

Fig. 3. Probability of outage versus transmitted power for clear weather, strong turbulence model $\left(\sigma_{\mathrm{R}}^{2}=1\right), \sigma_{s} / a=3$, and $R_{0}=0.5$ bits/chan. use.

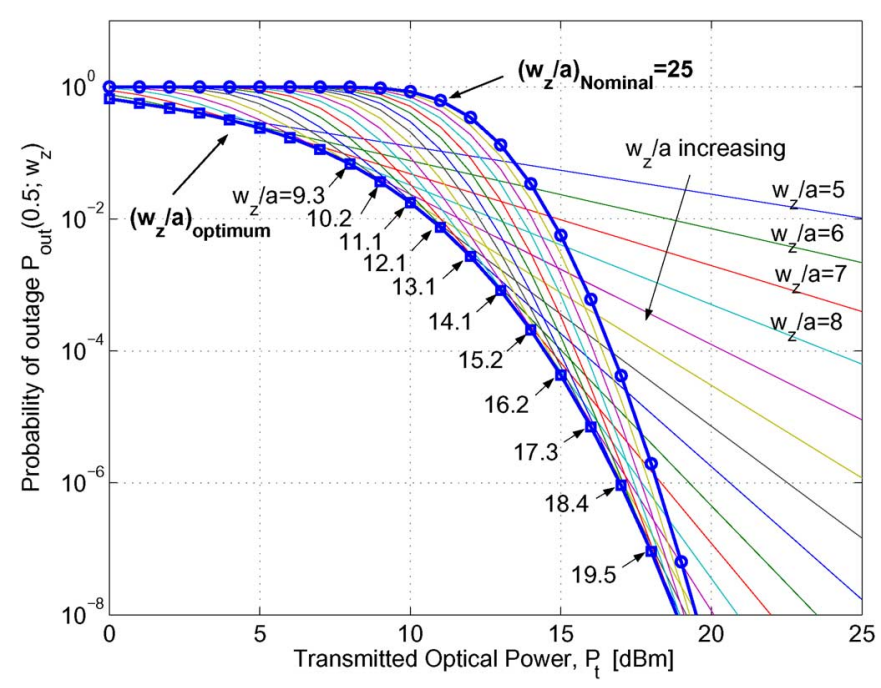

Fig. 4. Probability of outage versus transmitted power for light fog, weak turbulence model $\left(\sigma_{\mathrm{R}}^{2}=0.1\right), \sigma_{s} / a=3$, and $R_{0}=0.5$ bits/chan. use.

The probability of outage for the light fog case is computed using the weak fading model (18) and is presented in Fig. 4. The code rate is fixed at $R_{0}=0.5$ (bits/channel use) and $w_{z} / a$ varied from 5 to 25 . From the figure, link margins of 3- and 2-dB optical are obtained at $P_{\text {out }}=10^{-3}$ and $10^{-4}$, respectively. The behavior of this system is in contrast to the strong fading case discussed earlier. The optimum beam width changes rapidly with $P_{t}$, as shown in Fig. 4, where the corresponding values for the optimum beam widths $w_{z}^{\text {opt }} / a$ are presented. This increased sensitivity to misalignment fading can be justified due to the weak fading and high attenuation in this channel. Qualitatively, increases in $P_{t}$ are traded off for increases in the beam width to mitigate the impact of pointing errors. Transmitters designed for these channels need to have accurate control of their beam widths as significant gains can be made with the proper selection of $w_{z} / a$. 


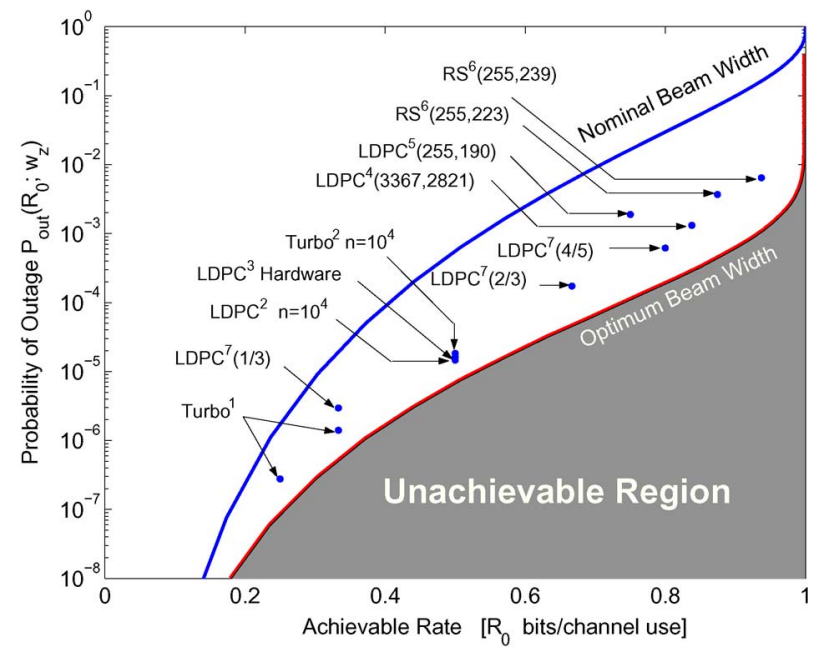

Fig. 5. Probability of outage versus achievable rate for nominal and optimum beam widths for light fog $\left(\sigma_{\mathrm{R}}^{2}=0.1\right), \sigma_{s} / a=3$ and $P_{t}=16 \mathrm{dBm}$. A variety of $(n, k)$ error control codes of rate $k / n$ are applied to this channel, and the performance presented at BER $=10^{-6}$. Hardware implemented Turbo ${ }^{1}$ codes with $R_{0}=\{1 / 4,1 / 3\}$ and $k=8920$ [30]. LDPC $^{2}$ and Turbo ${ }^{2}$ with rate $1 / 2$ and $n=10^{4}$ [31]. Hardware implementation for $\mathrm{LDPC}^{3}$ with $R_{0}=1 / 2$ and $k=4096$ [32]. $\mathrm{LDPC}^{4}$ [33] and $\mathrm{LDPC}^{5}$ of code rate 0.75 [34]. High-rate Reed-Solomon codes $\mathrm{RS}^{6}$ with $R_{0}=0.874$ and 0.937 [35]. LDPC ${ }^{7}$ with $k=1024$ [36].

\section{Probability of Outage and Achievable Rates}

In this section, the tradeoff between the $P_{\text {out }}$ and the maximum achievable rate is analyzed for optimum and nominal beam widths. The light fog case is considered, and $P_{t}, \sigma_{s}^{2}$ are fixed to the nominal values in Table III. The $P_{\text {out }}$ versus $R_{0}$ tradeoff is governed by (18) and parameterized by the beam width $w_{z}$. For a given $R_{0}, w_{z}^{\text {opt }}$ minimizes $P_{\text {out }}\left(R_{0} ; w_{z}^{\text {opt }}\right)$. Fig. 5 illustrates the tradeoff relation for optimum beam width $P_{\text {out }}\left(R_{0} ; w_{z}^{\text {opt }}\right)$ and fixed nominal beam width. Note that the value of $w_{z}^{\text {opt }}$ varies for each point on the curve.

As discussed in Section IV-B, not all pairs $\left(P_{\text {out }}, R_{0}\right)$ are achievable, resulting in an unachievable region. For pairs in the unachievable region, reliable communication is not possible. Thus, the $P_{\text {out }}\left(R_{0} ; w_{z}^{\text {opt }}\right)$ curve is the optimum tradeoff between outage probability and achievable rate for the given weather conditions. It is clear from Fig. 5 that for a given probability of outage, there is a significant gain in the achievable rate when utilizing the optimum beam over the nominal beam. For example, if the system is designed to meet $P_{\text {out }}=10^{-5}$, then the maximum code rate that can be reliably transmitted over this channel using the nominal beam width is $R_{0}=0.3$, while when utilizing the optimum beam, $R_{0}=0.54$, which is an increase of $80 \%$ in the achievable rate.

Although beam width optimization increases $R_{0}$, these achievable rates represent the maximum rates for which reliable communications is possible. Error control codes must be applied to approach these $R_{0}$ values with practical complexity. Well-known error control codes with $k$ bits per code word and block length $n$ are applied to the channel, and their performance is plotted in Fig. 5. For the simulations, an outage was defined as the event that the decoded BER $>10^{-6}$. The SNR corresponding to BER $=10^{-6}$ was found for each code through simulation and, via (3), the corresponding $h_{0}$ was computed. Substituting $h_{0}$ and the $w_{z}^{\text {opt }}$ for the code rate $R_{0}=$ $k / n$ into (18) gives the probability of outage. At low rates, hardware-based Turbo codes for space communications [30], as well as low-density parity-check (LDPC) [31] codes with large $n$, approach the optimal $P_{\text {out }}\left(R_{0} ; w_{z}^{\text {opt }}\right)$. At higher rates, Reed-Solomon [35] and low-complexity LDPC codes for fiber optical applications [33], [34], [36] can also be designed to operate close to the $P_{\text {out }}\left(R_{0} ; w_{z}^{\text {opt }}\right)$ curve. Of notable interest is the performance using a hardware-implemented rate-1/2 LDPC code [32]. From Fig. 5, the code achieves a $P_{\text {out }}=1.8 \times 10^{-5}$, while the optimum rate is $R_{0}=0.575$. Thus, this practical code can realize approximately $87 \%$ of the maximum rate. Significant gains in achievable rate are available by beam optimization, and the optimal tradeoff between $P_{\text {out }}$ and $R_{0}$ derived here can be used as a design guide when selecting code rates in practical FSO channels.

\section{Conclusion}

This paper considers the design of FSO channels corrupted by atmospheric turbulence and pointing errors from an information theory perspective. New statistical models are presented where beam width, pointing error, and detector size are considered. These models are used to derive fundamental limits on outage probability and achievable rates for FSO channels. In strong turbulence channels, a link margin gain of $5 \mathrm{~dB}$ is obtained over a nominal beam width by optimizing the beam width for a fixed code rate. For fixed transmitted power, using the optimum beam width gives large gains in achievable rates. At $P_{\text {out }}=10^{-5}$, the achievable rate in light fog is increased by $80 \%$ through beam optimization. Error-correcting codes are then applied, and a previously reported hardware implementation of an LDPC code can achieve $87 \%$ of the maximum rate at $P_{\text {out }}=1.8 \times 10^{-5}$.

It has been demonstrated that optimization of the beam width leads to significant increases in the channel capacity subject to outage. Furthermore, most of the achievable rate can be realized using realistic and practical error-correcting codes. Thus, this paper is a design guide for FSO communication systems, which allows for the optimization of channel capacity over a variety of weather conditions and for the selection of code rate at a given probability of outage.

\section{APPENDIX \\ Misalignment FADing $h_{\mathrm{p}}(r)$ ApProximation}

Consider approximating the integration in (8) by an integration over a square of equal area to the detector, i.e., with side length $\sqrt{\pi} a$. It follows that (8) can be approximated as

$$
h_{\mathrm{p}}(r) \approx \int_{-\sqrt{\pi} a / 2}^{\sqrt{\pi} a / 2} \frac{\sqrt{2} E}{\sqrt{\pi w_{z}^{2}}} \exp \left(-\frac{2\left(x^{\prime}-r\right)^{2}}{w_{z}^{2}}\right) d x^{\prime}
$$

where $E=\operatorname{erf}\left(\sqrt{\pi} a / \sqrt{2} w_{z}\right)$, and $\operatorname{erf}(x)=(2 / \sqrt{\pi}) \int_{0}^{x} e^{-u^{2}} d u$ is the error function. Expanding the exponential term into its 


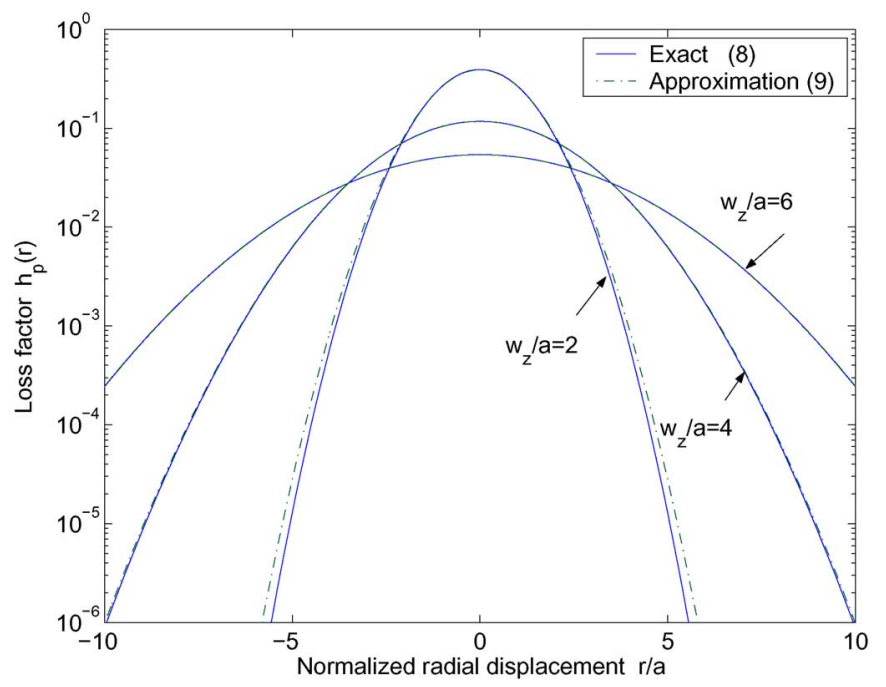

Fig. 6. Exact and approximate values of $h_{\mathrm{p}}(r)$ for different values of $w_{z} / a$.

Taylor series, integrating and simplifying results in

$$
\begin{aligned}
h_{\mathrm{p}}(r) \approx \frac{\sqrt{2} a E}{w_{z}}+ & \frac{2 E}{\sqrt{\pi}} \sum_{\substack{m=3 \\
\text { odd even }}}^{\infty} \sum_{\substack{\ell=0 \\
\text { even }}}^{m} \frac{(-1)^{(m-1) / 2}}{(m)\left(\frac{m-1}{2}\right) !} \\
& \times\left(\begin{array}{c}
m \\
\ell
\end{array}\right)\left(\sqrt{2} \frac{r}{w_{z}}\right)^{\ell}\left(\frac{\sqrt{\pi} a}{\sqrt{2} w_{z}}\right)^{m-\ell} .
\end{aligned}
$$

In addition, (A-1) can be rearranged with respect to $\ell$ and written in the compact form

$$
h_{\mathrm{p}}(r) \approx \sum_{\substack{\ell=0 \\ \text { even }}}^{\infty} A_{\ell}\left(\frac{\sqrt{2} r}{w_{z}}\right)^{\ell}
$$

where $A_{\ell}$ 's are given by

$$
A_{\ell}=\frac{2 E}{\sqrt{\pi}} \sum_{\substack{m=\ell+1 \\
\text { odd }}}^{\infty} \frac{(-1)^{(m-1) / 2}}{(m)\left(\frac{m-1}{2}\right) !}\left(\begin{array}{c}
m \\
\ell
\end{array}\right)\left(\frac{\sqrt{\pi} a}{\sqrt{2} w_{z}}\right)^{m-\ell} .
$$

Defining $v=(\sqrt{\pi} a) /\left(\sqrt{2} w_{z}\right)$ and simplifying gives

$$
A_{0}=[\operatorname{erf}(v)]^{2}, \quad A_{2}=\frac{-2}{\sqrt{\pi}} \operatorname{erf}(v)\left[v \exp \left(-v^{2}\right)\right] .
$$

Equating the first two terms of the Taylor expansion of a Gaussian pulse to the same terms in (A-2) gives (9)

$$
h_{\mathrm{p}}(r) \approx A_{0} \exp \left(-\frac{2 r^{2}}{w_{z_{\mathrm{eq}}}^{2}}\right)
$$

where $w_{z_{\mathrm{eq}}}^{2}=w_{z}^{2} A_{0} /\left|A_{2}\right|$. The exact (8) and the approximate (9) expressions for $h_{\mathrm{p}}(r)$ are plotted in Fig. 6 for a variety of $w_{z}$. Notice that they are in close agreement for $w_{z} / a>6$, where NMSE $<10^{-3}$, as given in Table I.

\section{REFERENCES}

[1] D. J. Heatley, D. R. Wisely, I. Neild, and P. Cochrane, "Optical wireless: The story so far," IEEE Commun. Mag., vol. 36, no. 12, pp. 72-82, Dec. 1998.

[2] S. Karp, R. Gagliardi, S. Moran, and L. Stotts, Optical Channels. New York: Plenum, 1988.

[3] L. C. Andrews, R. L. Phillips, C. Y. Hopen, and M. A. Al-Habash, "Theory of optical scintillation," J. Opt. Soc. Amer. A, Opt. Image Sci., vol. 16, no. 6, pp. 1417-1429, Jun. 1999.

[4] M. A. Al-Habash, L. C. Andrews, and R. L. Philips, "Mathematical model for the irradiance probability density function of a laser propagating through turbulent media," Opt. Eng., vol. 40, no. 8, pp. 1554-1562, Aug. 2001.

[5] J. D. Barry and G. S. Mecherle, "Beam pointing error as a significant parameter for satellite borne, free-space optical communication systems," Opt. Eng., vol. 24, no. 6, pp. 1049-1054, Nov. 1985.

[6] C. C. Chen and C. S. Gardner, "Impact of random pointing and tracking errors on the design of coherent and incoherent optical intersatellite communication links," IEEE Trans. Commun., vol. 37, no. 3, pp. 252-260, Mar. 1989.

[7] S. Arnon and N. S. Kopeika, "Laser satellite communication networkvibration effect and possible solutions," Proc. IEEE, vol. 85, no. 10, pp. 1646-1661, Oct. 1997.

[8] S. Arnon, "Effects of atmospheric turbulence and building sway on optical wireless communication systems," Opt. Lett., vol. 28, no. 2, pp. 129-131, Jan. 2003.

[9] D. Kedar and S. Arnon, "Optical wireless communication through fog in the presence of pointing errors," Appl. Opt., vol. 42, no. 24, pp. 49464954, Aug. 2003.

[10] S. Arnon, "Optimization of urban optical wireless communication systems," IEEE Trans. Commun., vol. 2, no. 11, pp. 626-629, Nov. 2003.

[11] J. A. Anguita, I. B. Djordjevic, M. Neifeld, and B. V. Vasic, "Shannon capacities and error-correction codes for optical atmospheric turbulent channels," OSA J. Opt. Netw., vol. 4, no. 9, pp. 586-601, Sep. 2005.

[12] S. Haas and J. H. Shapiro, "Capacity of wireless optical communications," IEEE J. Sel. Areas Commun., vol. 21, no. 8, pp. 1346-1357, Oct. 2003.

[13] L. Jing and M. Uysal, "Optical wireless communications: System model, capacity and coding," in Proc. IEEE 58th Veh. Technol. Conf., Orlando, FL, Oct. 2003, pp. 168-172.

[14] X. Zhu and J. Kahn, "Free space optical communication through atmospheric turbulence channels," IEEE Trans. Commun., vol. 50, no. 8, pp. 1293-1300, Aug. 2002.

[15] X. Zhu and J. M. Kahn, "Performance bounds for coded free-space optical communications through atmospheric turbulence," IEEE Trans. Commun., vol. 51, no. 8, pp. 1233-1239, Aug. 2003.

[16] M. Uysal, J. Li, and M. Yu, "Error rate performance analysis of coded free-space optical links over gamma-gamma atmospheric turbulence channels," IEEE Trans. Wireless Commun., vol. 5, no. 6, pp. 1229-1233, Jun. 2006.

[17] E. Biglieri, J. Proakis, and S. Shamai, "Fading channels: Informationtheoretic and communications aspects," IEEE Trans. Inf. Theory, vol. 44, no. 6, pp. 2619-2692, Oct. 1998.

[18] C. E. Shannon, "A mathematical theory of communication," Bell Syst. Tech. J., vol. 27, pp. 379-423, Jul./Oct. 1948.

[19] R. J. Hill and R. G. Frehlich, "Probability distribution of irradiance for the onset of strong scintillation," J. Opt. Soc. Amer. A, Opt. Image Sci., vol. 14, no. 7, pp. 1530-1540, Jul. 1997.

[20] S. M. Flatte, C. Bracher, and G. Y. Wang, "Probability density functions of irradiance for waves in atmospheric turbulence calculated by numerical simulations," J. Opt. Soc. Amer. A, Opt. Image Sci., vol. 11, no. 7, pp. 2080-2092, Jul. 1994.

[21] M. A. Naboulsi, H. Sizun, and F. de Fornel, "Fog attenuation prediction for optical and infrared waves," Opt. Eng., vol. 43, no. 2, pp. 319-329, Feb. 2004.

[22] I. Kim, B. McArthur, and E. Korevaar, "Comparison of laser beam propagation at $785 \mathrm{~nm}$ and $1550 \mathrm{~nm}$ in fog and haze for optical wireless communications," Proc. SPIE, vol. 4214, pp. 26-37, Feb. 2001.

[23] S. Muhammad, P. Köhldorfer, and E. Leitgeb, "Channel modeling for terrestrial FSO links," in Proc. IEEE Int. Conf. Transparent Opt. Netw., Barcelona, Spain, 2005, pp. 407-410.

[24] D. Bushuev and S. Arnon, "Analysis of the performance of a wireless optical multi-input to multi-output communication system," J. Opt. Soc. Amer. A, Opt. Image Sci., vol. 23, no. 7, pp. 1722-1730, Jul. 2006.

[25] B. E. A. Saleh and M. C. Teich, Fundamentals of Photonics. New York: Wiley, 1991.

[26] J. C. Ricklin and F. M. Davidson, "Atmospheric turbulence effects on a partially coherent Gaussian beam: Implications for free space laser 
communication," J. Opt. Soc. Amer. A, Opt. Image Sci., vol. 19, no. 9, pp. 1794-1802, Sep. 2002.

[27] L. C. Andrews, Special Functions of Mathematics for Engineers. New York: McGraw-Hill, 1992

[28] E. Korevaar, I. I. Kim, and B. McArthur, "Atmospheric propagation characteristics of highest importance to commercial free space optics," Proc. SPIE, vol. 4976, pp. 1-12, Apr. 2003.

[29] Maxim Integrated Products, Inc., Transimpedance Amplifier MAX3744, 2006. Data sheet. [Online]. Available: http://www.maxim-ic.com

[30] The Consultive Committee for Space Data Systems (CCSDS), TM Synchronization and Channel Coding, Jun. 2006. Informational Rep., Issue 1: Green Book. [Online]. Available: http://public.ccsds.org/

[31] T. J. Richardson, M. A. Shokrollahi, and R. L. Urbanke, "Design of capacity-approaching irregular low-density parity-check codes," IEEE Trans. Inf. Theory, vol. 47, no. 2, pp. 619-637, Feb. 2001.

[32] T. Richardson and R. Urbanke, "The renaissance of Gallager's lowdensity parity-check codes," IEEE Commun. Mag., vol. 41, no. 8, pp. 126131, Aug. 2003.

[33] I. B. Djordjevic, S. Sankaranarayanan, and B. Vasic, "Irregular lowdensity parity-check codes for long-haul optical communications," IEEE Photon. Technol. Lett., vol. 16, no. 1, pp. 338-340, Jan. 2004.

[34] I. B. Djordjevic, S. Sankaranarayanan, and B. Vasic, "Projective-plane iteratively decodable block codes for WDM high-speed long-haul transmission systems," J. Lightw. Technol., vol. 22, no. 3, pp. 695-702, Mar. 2004.

[35] S. Lin and D. J. Costello, Error Control Coding. Englewood Cliffs, NJ: Prentice-Hall, 2004

[36] D. Divsalar, S. Dolinar, and C. Jones, "Low-rate LDPC codes with simple protograph structure," in Proc. IEEE Int. Symp. Inform. Theory, Adelaide, Australia, Sep. 4-9, 2005, pp. 1622-1626.

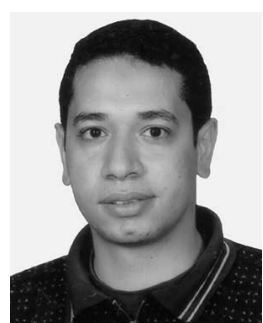

Ahmed A. Farid (S'06) received the B.S. and M.S. degrees from the University of Alexandria, Alexandria, Egypt, in 1996 and 2000, respectively, and the M.A.Sc. degree from McMaster University, Hamilton, ON, Canada, in 2004, where he is currently working toward the Ph.D. degree.

His current research interests are in wireless optical communications, coding, and modulation for free-space optical links.

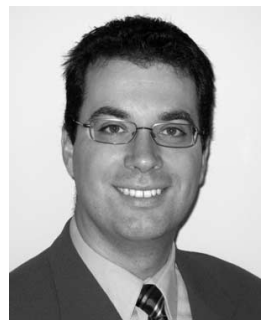

Steve Hranilovic (S'94-M'03) received the B.A.Sc. degree (with honors) in electrical engineering from the University of Waterloo, Waterloo, ON, Canada, in 1997 and the M.A.Sc. and Ph.D. degrees in electrical engineering from the University of Toronto, Toronto, ON, Canada, in 1999 and 2003, respectively.

He is an Assistant Professor with the Department of Electrical and Computer Engineering, McMaster University, Hamilton, ON. His research interests are in the areas of free-space and wireless optical communications, digital communications algorithms, and electronic and photonic implementation of coding and communication algorithms. He is the author of the book Wireless Optical Communications Systems (New York: Springer, 2004).

Dr. Hranilovic was awarded the Government of Ontario Early Researcher Award in 2006. 